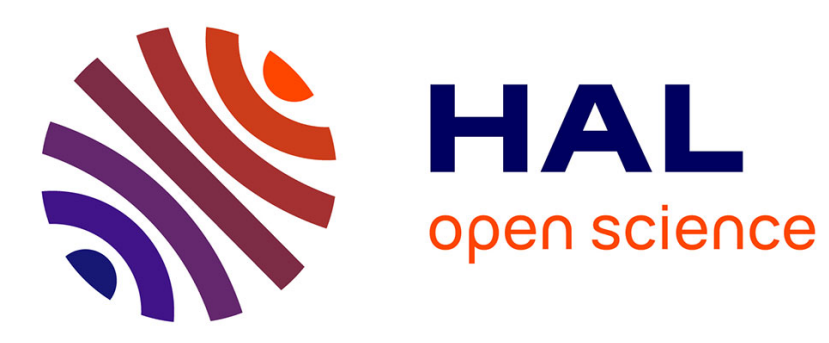

\title{
3D-2D ultrasound feature-based registration for navigated prostate biopsy: A feasibility study
}

Sonia y Selmi, Emmanuel Promayon, Jocelyne Troccaz

\section{To cite this version:}

Sonia y Selmi, Emmanuel Promayon, Jocelyne Troccaz. 3D-2D ultrasound feature-based registration for navigated prostate biopsy: A feasibility study. 38th Annual International Conference of the IEEE Engineering in Medicine and Biology Society (EMBC'16), Aug 2016, Orlando, FL, United States. pp.4109 - 4112, 10.1109/EMBC.2016.7591630 . hal-01387243

\section{HAL Id: hal-01387243 \\ https://hal.science/hal-01387243}

Submitted on 25 Oct 2016

HAL is a multi-disciplinary open access archive for the deposit and dissemination of scientific research documents, whether they are published or not. The documents may come from teaching and research institutions in France or abroad, or from public or private research centers.
L'archive ouverte pluridisciplinaire HAL, est destinée au dépôt et à la diffusion de documents scientifiques de niveau recherche, publiés ou non, émanant des établissements d'enseignement et de recherche français ou étrangers, des laboratoires publics ou privés. 


\title{
3D-2D Ultrasound Feature-Based Registration for Navigated Prostate Biopsy: a Feasibility Study
}

\author{
Sonia Y. Selmi ${ }^{1}$, Emmanuel Promayon ${ }^{1}$ and Jocelyne Troccaz ${ }^{1}$
}

\begin{abstract}
The aim of this paper is to describe a 3D-2D ultrasound feature-based registration method for navigated prostate biopsy and its first results obtained on patient data. A system combining a low-cost tracking system and a 3D2D registration algorithm was designed. The proposed 3D2D registration method combines geometric and image-based distances. After extracting features from ultrasound images, 3D and $2 \mathrm{D}$ features within a defined distance are matched using an intensity-based function. The results are encouraging and show acceptable errors with simulated transforms applied on ultrasound volumes from real patients.
\end{abstract}

\section{INTRODUCTION}

Prostate cancer is the second most common cancer worldwide for males [1]. Prostate biopsy procedures, performed to obtain and analyze tissue samples of the gland, are required for diagnosis. The clinical standard protocol is currently performed under UltraSound (US) control following a systematic protocol. Unfortunately, prostate cancer diagnosis is complicated by the lack of image quality and the low intrinsic contrast between tumor and non-tumor on US images

Conventional biopsies are performed under TRansrectal UltraSound (TRUS) guidance (see fig. 1). In clinical practice, a 12-core biopsy protocol is usually performed. These 12 samples have to be well-distributed and located in 12 different 3D anatomical regions of the prostate in order to obtain a reliable cancer diagnosis. Precisely localizing the biopsy sites is challenging because the gland has a symmetric shape and because the prostate moves and is deformed by the patient motion and the TRUS probe pressure.

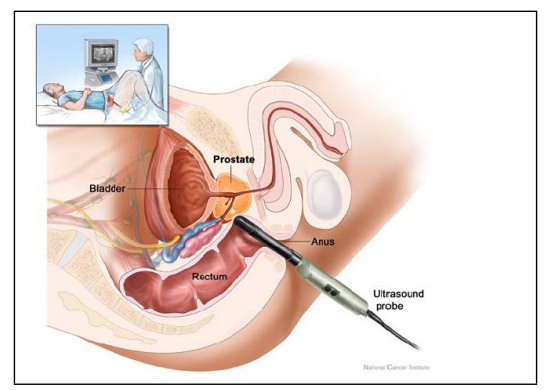

Fig. 1. TRUS-guided procedure (Image from Terese Winslow, the National Cancer Institute website).

Assisted prostate biopsy has been widely studied over the last fifteen years in order to mitigate the presented problems. Its objectives are to locate the exact position of the samples

*This work was supported by the French Single Inter-Ministry Fund (FUI) MIRAS and ANR (French National Research Agency) grant CAMI labex ANR-11-LABX-0004

${ }^{1}$ Univ. Grenoble Alpes / CNRS / TIMC-IMAG UMR 5525, F-38041, France Email: FirstName.Namedimag.fr and to guide the clinician accurately to specific targets. The clinically available systems mainly combine the ability to track the US probe, using a robot [2] or a localizer [3], [4], to the ability to track the prostate from the image information [5].

Various approaches were investigated to register TRUS images during biopsy procedure to an initially acquired 3D image (see Table I for a short bibliography comparison).

TABLE I

MAIN LITERATURE OF US REGISTRATION METHODS FOR PROSTATE BIOPSY. EM : ELECTROMAGNETIC

\begin{tabular}{|c|c|c|c|}
\hline Reference & $\begin{array}{c}\text { Tracking } \\
\text { system }\end{array}$ & Registration & Real-time \\
\hline $\begin{array}{c}\text { Xu et al. } \\
\text { [3], [6] }\end{array}$ & EM & $\begin{array}{c}\text { 2.5D-3D US, Iconic, } \\
\text { Rigid }\end{array}$ & yes \\
\hline $\begin{array}{c}\text { Baumann } \\
\text { et al. [5] }\end{array}$ & no & $\begin{array}{c}\text { 3D-3D US, Iconic, } \\
\text { Elastic \& Rigid }\end{array}$ & no \\
\hline $\begin{array}{c}\text { De Silva } \\
\text { et al. [4] }\end{array}$ & EM & $\begin{array}{c}\text { 2D-3D US, Iconic, } \\
\text { Rigid }\end{array}$ & yes \\
\hline $\begin{array}{c}\text { Khallaghi } \\
\text { et al. [9] }\end{array}$ & EM & $\begin{array}{c}\text { 2D-3D US, Iconic, } \\
\text { Rigid \& FE Model }\end{array}$ & no \\
\hline
\end{tabular}

The method presented by Xu et al. [3], [6] is based on realtime 2D TRUS - MR images fusion using a electromagnetic tracking and intraoperative image registration to superimpose MR image on US image, without taking prostate deformation into account. Baumann et al. [7] proposed non rigid registration of 3D US volumes for accurate estimation of prostate motion and deformation. However, the tracking is not performed in real-time due to long $3 \mathrm{D}$ volume acquisition time and computing time. The average accuracy measured on patient data sets was however submillimetric.

In 2013, De Silva et al. [4] proposed a 2D-3D rigid US registration to compensate for prostate motion with a measured accuracy of less than $2 \mathrm{~mm}$ and a speed of $1.1 \mathrm{~s}$. In [8], the same authors improved their method using a prior model of probe-prostate motion. In [9], Khallaghi et al. presented a 2D-3D US registration by reconstructing a thin-volume around the real-time 2D US imaging plane. They applied a rigid registration and FEM-based technique for deformation and obtained a mean target registration error (TRE) of $3.15 \mathrm{~mm}$, although not in real-time.

This paper describes a feasibility study for navigated prostate biopsies combining a low-cost tracking system and a 2D-3D US registration method. The general framework of this newly developed 2D/3D registration aims to provide accurate navigation in between two precise 3D/3D registra- 
tion steps performed using the method presented in [7]. In this new approach, we propose to use a low-cost inertial sensor to track the US probe. Inertial sensors are known to be inaccurate because of noise and drift effects. Our method takes this inaccuracy into account. Real-time 2D US slices are rigidly registered with a reference TRUS volume, using the spatial transform provided by the sensor as an initial pose estimation. The reference volume is acquired at the start of the biopsy procedure or at regular time interval when a 3D/3D registration is performed. The presented 2D/3D registration is an hybrid method that couples geometric and image-based registration. We evaluated the performance of the registration algorithm on patient data in terms of accuracy and speed. This paper presents an initial evaluation of this method.

\section{MATERiAls AND METHOD}

This section describes the three steps of our registration method: a) feature extraction from 2D and 3D local intensity, b) feature description using voxel-based information and c) matching of nearby 2D-3D features in order to compute the spatial transform between images. A workflow is presented in Fig. 2.

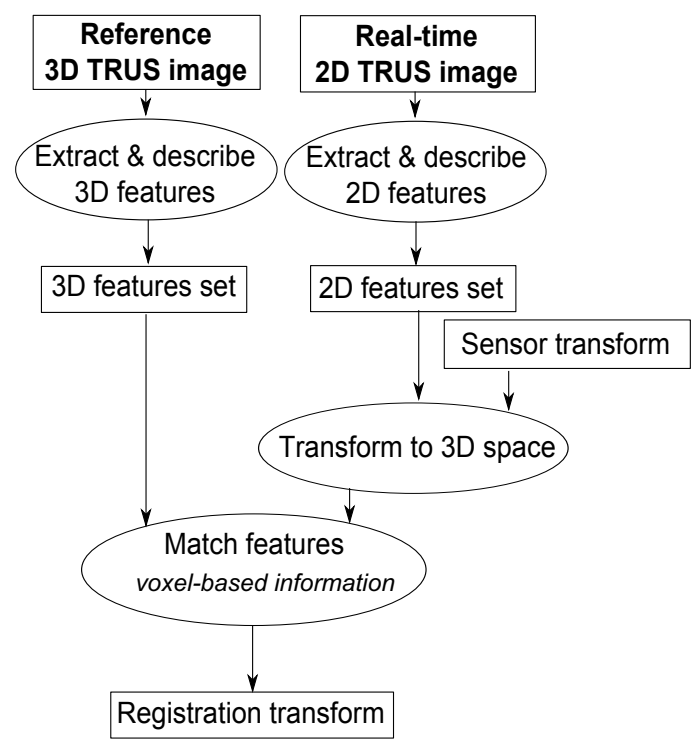

Fig. 2. Workflow of our algorithm

\section{A. Feature extraction}

The first step consists in extracting interest points from the 2D and 3D US images. We used the Hessian detector to detect bidimensional (in 2D) or tridimensional (in 3D) significant intensity changes. It is invariant to image rotation. In 2D, the Hessian matrix is a square matrix of second-order partial derivatives:

$$
H(\mathbf{x})=\left[\begin{array}{ll}
L_{x x}(\mathbf{x}) & L_{x y}(\mathbf{x}) \\
L_{x y}(\mathbf{x}) & L_{y y}(\mathbf{x})
\end{array}\right]
$$

where $L_{x x}(\mathbf{x})$ is second partial derivative in the $x$ direction and $L_{x y}(\mathbf{x})$ is second partial derivative in the $x$ and $y$ direction. We used derivatives of the image smoothed by a Gaussian kernel: $L(\mathbf{x})=g\left(\sigma_{I} \otimes I(\mathbf{x})\right)$. A corner measure is defined [10] as follow :

$$
R=\operatorname{det}(H)-\alpha \operatorname{trace}^{2}(H), 0.04 \leqslant \alpha \leqslant 0.06
$$

It detects blob-like structures at locations where the determinant is maximum. This measure penalizes longer structures that have small second derivatives in a single direction [11]. In order to reduce the computational cost, only the $N_{\mathrm{R}}^{2 \mathrm{D}}$ features with the highest $R$ values are kept.

The same principles are applied to compute and select the $N_{\mathrm{R}}^{3 \mathrm{D}}$ features in the $3 \mathrm{D}$ images using a 3D Hessian matrix.
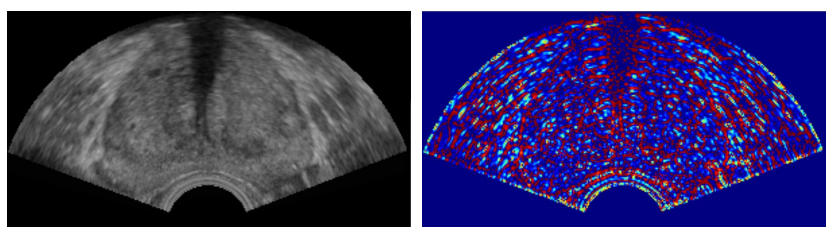

(a)

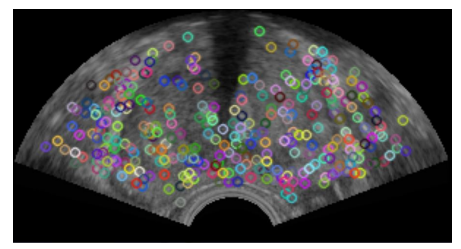

(b)

Fig. 3. (a) 2D US image (left) and its Hessian response map (right), high values are in red, small in blue. (b) Resulting features with the highest $R$.

\section{B. Feature description}

Once the features are extracted from the images, the next step consists in associating a descriptor to each feature. The feature description is required to perform discriminative matching. To identify both 2D and 3D features, an intensitybased description is build using the pixel or voxel intensity and the mean intensity and intensity histogram computed in a given neighborhood of the feature.

\section{Matching}

In order to compute the rigid transform between a given $2 \mathrm{D}$ image and 3D volume, our matching algorithm minimizes the distance between the positions of the 2D feature and the positions of the 3D features using the least square method. The Iterative Closest Points (ICP) algorithm [12] is widely used for geometric alignment of 3D models when an initial estimate of the relative pose is known. It iteratively refines the transform by repeatedly generating pairs of corresponding features and minimizing an error metric, generally an Euclidian distance.

The four steps of our matching algorithm are illustrated in Fig. 4.

1) Initial transform: The first step consists in initializing the algorithm using the $T_{\text {sensor }}$ transform given by the inertial sensor. This transform gives an initial mapping of the $2 \mathrm{D}$ feature set $\mathbf{F}_{2 D}$ in the $3 \mathrm{D}$ world coordinate system. Each 2D features have an initial position in 3D. 
2) Inliers selection: At each iteration, candidates in the 3D feature set $\mathbf{F}_{3 D}$, named inliers, are selected by considering the closest geometric neighbors of the 2D features (i.e., all the 3D features that are under a given $d_{\text {inliers }}$ distance to a $2 \mathrm{D}$ feature). A $k$ - $d$ tree is used to accelerate this selection. The distance threshold $d_{\text {inliers }}$ is initialized at the first iteration and is then decreased at each iteration in function of the minimization error obtained at the end of the iteration. This step optimizes the minimization process and improve the convergence of the ICP algorithm.
(1)

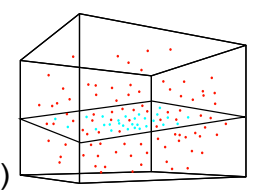

(3)

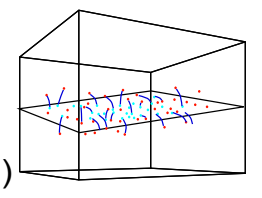

(2)

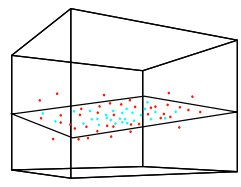

(4)

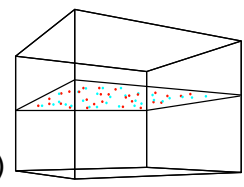

Fig. 4. (1) Initial transform (2) Inlier's selection (3) Point matching (4) Minimization

3) Voxel-based features matching: The next stage of the ICP algorithm is to associate a $2 \mathrm{D}$ feature to one and only one $3 \mathrm{D}$ feature. It combines the geometric pre-selection with an intensity-based distance $d\left(f_{1}, f_{2}\right)$. For each feature $f_{2 D} \in \mathbf{F}_{2 D}$, the $n_{n}$ geometrically closest $3 \mathrm{D}$ features are selected. For each of them, $d\left(f_{2 D}, f_{3 D}\right)$ is computed and the $3 \mathrm{D}$ feature which minimizes this distance is paired to the considered 2D feature.

The distance $d\left(f_{1}, f_{2}\right)$ combines three terms based on the local intensity of the features in order to get reliable pairing. $d\left(f_{1}, f_{2}\right)$ is defined as follows:

$$
\mathbf{d}\left(f_{1}, f_{2}\right)=\alpha_{1} d_{1}+\alpha_{2} d_{2}+\alpha_{3} d_{3},
$$

where: $\sum \alpha_{i}=1 . d_{1}$ compares the mean intensity in the neighborhood of the features:

$$
d_{1}\left(\mathbf{I}_{1}, \mathbf{I}_{2}\right)=\frac{\left\|\overline{\mathbf{I}}_{1}-\overline{\mathbf{I}}_{2}\right\|}{256}
$$

$d_{2}$ uses the Bhattacharyya distance which quantifies the similarity of two normalized histograms:

$$
d_{2}\left(\mathbf{H}_{1}, \mathbf{H}_{2}\right)=\frac{\sum_{I}\left(\mathbf{H}_{1}-\overline{\mathbf{H}}_{1}\right)\left(\mathbf{H}_{2}-\overline{\mathbf{H}}_{2}\right)}{\sqrt{\sum_{I}\left(\mathbf{H}_{1}-\overline{\mathbf{H}}_{1}\right)^{2} \sum_{I}\left(\mathbf{H}_{2}-\overline{\mathbf{H}}_{2}\right)^{2}}}
$$

with

$$
\overline{\mathbf{H}}_{k}=\frac{1}{N} \sum_{J} \mathbf{H}_{k}(J)
$$

$\mathrm{N}$ is the total number of histogram bins.

And $d_{3}$ consists in a sum of squared distance:

$$
\mathbf{d}_{3}=\sqrt{\frac{1}{|\Omega|} \sum_{j \in \Omega}\left(I_{1}(j)-I_{2}(j)\right)^{2}}
$$

4) Updating the transform: The final step of ICP algorithm computes the transform which minimizes the geometric distance between paired features. [13] is used for direct leastsquare error minimization. This step is iterated until convergence or failure (when a maximum number of iterations is reached).

\section{VALIDATION}

\section{A. Data}

Patient data have been collected from the Urostation®system (Koelis®, France) and have been acquired during a prostate biopsy procedure using a US probe. Each dataset is composed of 3D volumes with $300 \times 159 \times 222$ voxels and a voxel size of $0.42 \mathrm{~mm} \times 0.42 \mathrm{~mm} \times 0.42 \mathrm{~mm}$.

\section{B. Parameter selection}

There are 6 parameters that control algorithm operation and performance:

- $N_{\mathrm{R}}^{2 \mathrm{D}}=300, N_{\mathrm{R}}^{3 \mathrm{D}}=20,000$

- $\alpha=0.05$

- window size : $5 \times 5$ pixels in $2 \mathrm{D}$ and $5 \times 5 \times 5$ voxels in $3 \mathrm{D}$

- $d_{\text {inliers }}=40$

- $\alpha_{1}=\alpha_{2}=\alpha_{3}=\frac{1}{3}$

- $n_{n}=5$

- ICP algorithm convergence: $r m s_{i-1}-r m s_{i}<0.01$ or $n b_{\text {iteration }}>150$

For this feasibility study, these parameters were empirically estimated to provide a tradeoff between computing time and accuracy.

\section{Registration accuracy under simulated rotation and translation}

The proposed 2D-3D registration method was evaluated on 5 patient datasets. For each dataset, a 2D slice was extracted from the 3D volume with known transforms. To simulate artificial rotations and translations mimicking the TRUS probe movements, these transforms were either a rotation ranging from $-15^{\circ}$ to $15^{\circ}$ (by steps of $1^{\circ}$ ) around each axis or a translation ranging from $-10 \mathrm{~mm}$ to $10 \mathrm{~mm}$ (by steps of $0.5 \mathrm{~mm}$ ) on each axis. Translations and rotations are separately tested. We then registered the transformed 2D slices to the 3D volume using our method and computed the resulting registration error. For each image, 4 different starting positions were used to simulate the 2D slice initial $T_{\text {sensor }}$ transform. A total 864 registrations per 3D volume were computed, for an overall total of 4320. RMS error is measured between ground truth 2D feature's position (i.e., the feature actual positions in the extracted 2D slice) and their position after registration.

Table II and III show the registration results. Rotation around $\mathrm{x}$-axis, $\mathrm{y}$-axis and $\mathrm{z}$-axis corresponds respectively to an out-of-plane rotation around the horizontal axis, an out-ofplane rotation around a vertical axis and an in-plane rotation. Figure 5 shows the variation of RMS error depending on the artificial translation and rotation. 
TABLE II

RESULTS OF REGISTRATION FOR ROTATION.

\begin{tabular}{c|c|c|c} 
RMS(mm) & $\begin{array}{c}\text { rotation } \\
\text { around x-axis }\end{array}$ & $\begin{array}{c}\text { rotation } \\
\text { around } \mathbf{y} \text {-axis }\end{array}$ & $\begin{array}{c}\text { rotation } \\
\text { around } \mathbf{z} \text {-axis }\end{array}$ \\
\hline Mean & 3.53 & 1.97 & 2.71 \\
\hline Std dev & 3.86 & 1.99 & 2.63 \\
\hline$<\mathbf{5 m m}$ & $74 \%$ & $88 \%$ & $80 \%$
\end{tabular}

TABLE III

RESULTS OF REGISTRATION FOR TRANSLATION.

\begin{tabular}{c|c|c|c} 
RMS(mm) & $\begin{array}{c}\text { translation } \\
\text { on } \mathbf{x} \text {-axis }\end{array}$ & $\begin{array}{c}\text { translation } \\
\text { on } \mathbf{y} \text {-axis }\end{array}$ & $\begin{array}{c}\text { translation } \\
\text { on } \mathbf{z} \text {-axis }\end{array}$ \\
\hline Mean & 2.23 & 1.73 & 2.74 \\
\hline Std dev & 2.21 & 1.62 & 2.75 \\
\hline$<\mathbf{5 m m}$ & $84 \%$ & $91 \%$ & $78 \%$
\end{tabular}

\section{Computing-time}

Running times are for a $\mathrm{C}++$ implementation running on a Intel(R) Core(TM) i7-3740QM CPU @ 2.70GHz processor. Each 2D-3D registration took less than 2 seconds (2D features extraction being faster than the matching procedure).

\section{DISCUSSION AND CONCLUSION}

We presented an initial feasibility study of a novel 2D3D feature-based registration method. The final objective is to perform real-time US rigid registration to follow the prostate during a freehand TRUS-guided prostate biopsy. The feature-based registration uses image intensity information to enhance point correspondence. These first results are encouraging in terms of computation time. Most of the 3D features were detected as $2 \mathrm{D}$ features, ensuring a good match between the detected structures. Next step will consist in registering data coming from two different $3 \mathrm{D}$ volumes of the same patient: slices should be extracted from one volume and registered to the other volume. This will also evaluate the clinical relevance in terms of rotation and translation ranges. It should allow us to assess the algorithm in presence of both noise in the images and prostate deformation between two acquisitions.

To improve our method, a multi-scale version of the detector can be implemented to extract features and also other similarity measure can be used to compare features. Besides, more experiments should be performed to estimate parameter selection and sensitivity on a large image database. Finally, decreasing the registration time can be accomplished with a GPU implementation.

\section{REFERENCES}

[1] R. Siegel, C. DeSantis, and A. Jemal. Colorectal cancer statistics, 2014. CA: A Cancer Journal for Clinicians, 64(2):104-117.

[2] J. Bax, D. Cool, L. Gardi, K. Knight, D. Smith, J. Montreuil, S. Sherebrin, C. Romagnoli, and A. Fenster. Mechanically assisted 3d ultrasound guided prostate biopsy system. Medical Physics, 35(12):5397-5410, December 2008.

[3] S. Xu, J. Kruecker, B. Turkbey, N. Glossop, A. Singh, P. Choyke, P. Pinto, and B. Wood. Real-time MRI-TRUS fusion for guidance of targeted prostate biopsies. Computer Aided Surgery, 13(5):255-264, Jan. 2008.

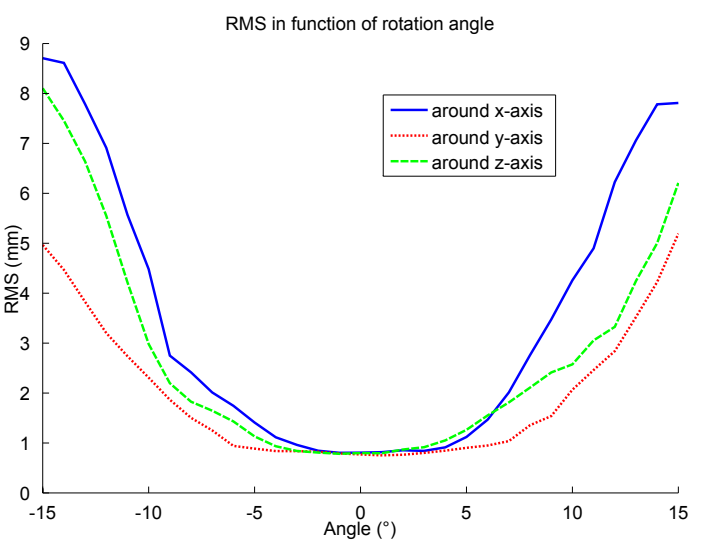

(a)

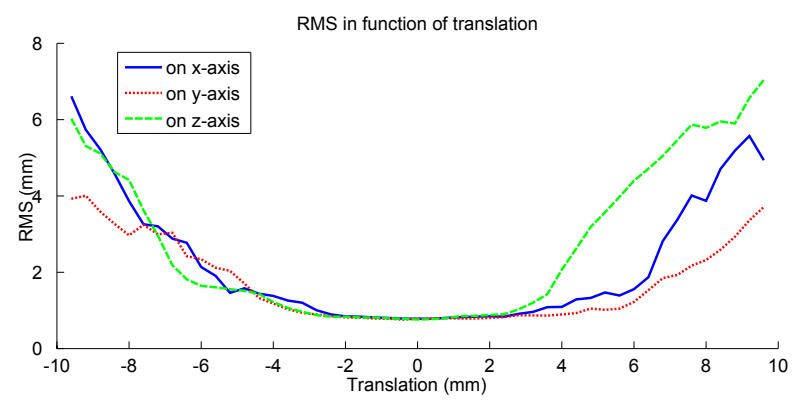

(b)

Fig. 5. (a) RMS error (mm) in function of rotation $\left({ }^{\circ}\right)$ (b) RMS error $(\mathrm{mm})$ in function of translation $(\mathrm{mm})$

[4] T. De Silva, A. Fenster, D. Cool, L. Gardi, C. Romagnoli, J. Samarabandu, and A. Ward. 2d-3d rigid registration to compensate for prostate motion during 3d TRUS-guided biopsy. Medical Physics, 40(2):022904, February 2013. MICCAI 2013, 8150, 124-131.

[5] M. Baumann, P. Mozer, V. Daanen, and J. Troccaz. Towards 3d Ultrasound Image Based Soft Tissue Tracking: A Transrectal Ultrasound Prostate Image Alignment System. MICCAI 2007, 4792, 26-33.

[6] S. Xu, J. Kruecker, P. Guion, N. Glossop, Z. Neeman, P. Choyke, A. Singh, and B. Wood. Closed-Loop Control in Fused MR-TRUS Image-Guided Prostate Biopsy. MICCAI 2007, 4791, 128-135.

[7] Baumann, M. and Mozer P. and Daanen V. and Troccaz J. Prostate biopsy tracking with deformation estimation. Medical Image Analysis 2011, 16, 562-576.

[8] T. De Silva, D. Cool, J. Yuan, C. Romognoli, A. Fenster, and A. Ward. Improving 2d-3d Registration Optimization Using Learned Prostate Motion Data.

[9] S. Khallaghi, C. Snchez, S. Nouranian, S. Sojoudi, S. Chang, H. Abdi, L. Machan, A. Harris, P. Black, M. Gleave, L. Goldenberg, S. Fels, and P. Abolmaesumi. A 2d-3d Registration Framework for Freehand TRUS-Guided Prostate Biopsy. MICCAI 2015, 9350, 272-279.

[10] C. Harris and M. Stephens. A combined corner and edge detector. pages 147-151, 1988.

[11] K. Mikolajczyk and C. Schmid. An Affine Invariant Interest Point Detector. ECCV 2002, 2350, 128-142.

[12] S. Rusinkiewicz and M. Levoy. Efficient variants of the ICP algorithm. In 3-D Digital Imaging and Modeling, pages 145-152, 2001.

[13] K.S. Arun, T.S. Huang, and S.D. Blostein. Least-Squares Fitting of Two 3-D Point Sets. Pattern Analysis and Machine Intelligence, PAMI-9(5):698-700, Sept.1987. 